\title{
Effects of Gravity on the Storage of Ultracold Neutrons
}

J M Pendlebury* and D J Richardson $\uparrow$

Physics building, University of Sussex, Brighton BN1 9QH, U K

Present addresses:

*Institut Laue-Langevin, BP 156, 38042 Grenoble, France

†Opto-electronics Research Centre, University of Southampton, Southampton $\mathrm{SO} 95 \mathrm{NH}, \mathrm{UK}$

\begin{abstract}
A general phase space approach is used to examine the influence of gravity on the storage of ultracoid neutrons. In particular, consideration is given to the effects on the mean free path between wall collisions and also on the extent to which the rate of loss of UCN in wall collisions can still be related to the mean free path. The implications for experiments to determine the neutron beta decay lifetime are discussed.
\end{abstract}

\section{Introduction}

Ultracold neutrons (UCN) which can be stored in material traps will generally have kinetic energies less than $340 \mathrm{neV}$ and speeds of less than $8 \mathrm{~m} / \mathrm{s}$. Recent experiments to determine the neutron beta decay lifetime using stored UCN have used walls made from aluminium [1], solid oxygen [2] and Fomblin oil [3,4]. The maximum UCN energies, which could be stored, were $54 \mathrm{neV}, 69 \mathrm{neV}$ and $106 \mathrm{neV}$ respectively. A neutron with a kinetic energy of $106 \mathrm{neV}$ has a speed of $4.52 \mathrm{~m} / \mathrm{s}$. A neutron, or any other object, rising vertically at this speed against gravity, will come to rest after rising 1.04 metres. It is evident that gravity has a significant effect on UCN moving in traps with vertical dimensions which are typically some tens of centimetres.

UCN can be contained by the walls if their kinetic energy $E$ is less than the real part $V$ of the mean Fermi potential $[5,6] v_{f}=V-i W$, where

$V=\frac{2 \pi \hbar^{2}}{m} \sum_{i} N_{i} b_{i} \quad$ and $\quad W=\frac{\hbar^{2}}{2 m} \sum_{i} N_{i} l_{i}$

and $N_{i}$ is the number density of nuclei of type $i$ in the material of the wall

8 June 1993

For Nuclear Instruments and Methods A 
surface and $b_{i}$ is their bound scattering length. Also, $l_{i}=\sigma_{i \text { tot }}(k) / k$ where $k$ is the neutron wave vector and $\sigma_{i}$ tot is the total loss cross-section for inelastic upscattering and nuclear absorbtion processes caused by nuclei of type $i$ in the wall surface where the UCN wave function penetrates a distance of a few nanometres. Upscattering is caused by the thermal motions of these nuclei, and when it occurs, the final kinetic energy is high compared with that of UCN and, as a result, the neutron is lost from the trap. Both the $b_{i}$ and the $l_{i}$ and hence $v$ and $W$ can be taken to be independent of the UCN speed. One case where small 'non-fatal' energy transfers to UCN may just be detectable arises when there are macrocopic vibrations of the trap walls caused by local machinery. [3,6]. However, with good control of the environment these energy changes can be made negligible. In this paper we will assume that all neutrons which survive in the trap have bounced perfectly elastically throughout the period of storage.

We begin with a monochromatic group of UCN. After entering and being sealed into a trap the individual members of the group will, within a few tens of seconds, spread uniformly over the phase space which is available within the constraints of energy conservation and the trap geometry. The time taken to arive at this uniformity in phase space is governed firstly, by the wall collision rate, which is typically $20 \mathrm{~s}^{-1}$ and given by $v / \lambda$ where $\lambda$ is the mean free path between wall collisions and $v$ the neutron speed. Secondly, it involves the probability per collision of significant non-specular reflection, which is usually a few percent even for polished surfaces. In the case of the liquid walls of reference [3] which are exceptionally smooth, an area of corrugated surface is built into the trap to ensure that the relaxation time for approaching uniformity is only a few seconds. If lifetimes arising from wall losses alone are a few thousand seconds, the assumption of a quasi-steady state uniform distribution of UCN in all the accessible phase space after a few tens of seconds of containment should be accurate to about 1 part in $10^{3}$. At this low level there are discrepancies in the anisotropy of the velocities which are brought about because the loss probabilities in UCN wall collisions are higher at larger angles of incidence. A steady state is reached in which there is a dynamic equilibrium between the rate of selection in angle and the opposing rate of randomisation due to the non-specular reflections.

Thus, in the absence of gravity the neutrons will soon become uniformly distributed throughout the volume and will acquire a high degree of isotropy in their velocity directions. The mean free path $\lambda$ against wall collisions, as deduced originally by Clausius, is then given by $\lambda=4 \mathrm{Vol} / \mathrm{A}_{\mathrm{w}}$ where $\mathrm{Vol}$ is the volume of the trap and $A_{w}$ is the total area of all the internal walls exposed to the UCN. Continuing with zero gravity, the neutrons can be taken to have the same speed $v(E)$ and the same kinetic energy $E$ in all parts of the trap. On reflecting from a wall a neutron has a probability of loss per collision, which, after averaging over all the angles of incidence for an isotropic distribution of velocities incident on a surface with an ideal step function profile [5] is given by the expression 
$\bar{\mu}(E)=2 f\left[\frac{V}{E} \arcsin \left(\sqrt{\frac{E}{V}}\right)-\sqrt{\frac{(V-E)}{E}}\right]$

where $f=W / V$. For cases where the density of the wall material rises more slowly at the surface, the $\bar{\mu}(E)$ functions deviate very little from this last expression $[7,8]$. On the other hand, inhomogenious distributions of impurities such as hydrogen can cause deviations at the level of tens of percent. If the trap walls are all of the same material, and still there is no gravity, the rate of loss of UCN through collisions per unit area is the same on all parts of the walls. Then the rate of change of the total number, $N(t)$ of UCN within the trap is

$\frac{d N}{d t}=-\frac{N \bar{\mu}(E) v(E)}{\lambda}-\frac{N}{\tau_{n}}$

where $\tau_{n}$ is the neutron beta decay lifetime. The result is a pure exponential decrease of $N$ with a decay time constant $\tau_{g r o s s}$ given by

$\frac{1}{\tau_{\text {gross }}}=\frac{1}{\tau_{\text {wail }}}+\frac{1}{\tau_{n}}$

where

$\frac{1}{\tau_{\text {waI1 }}}=\frac{v(E) \bar{\mu}(E)}{\lambda}$

and

$\lambda=\frac{4 \mathrm{Vol}}{\mathrm{A}_{\mathrm{H}}}$.

Also, in the absence of gravity, and when the wall losses remain small, a number of other considerations of practical interest are very straight forward. For example, on opening a connection between a trap full of UCN and one which is empty, the UCN will spread themselves uniformly within a few tens of seconds so that they apportion themselves between the traps in the ratio of the trap volumes. On the other hand, if the connected traps are at different heights and gravity is allowed for, this simple result no longer applies and the methods of the next section will be needed to calculate the apportionment. Another aspect of the gravity free result in equation (5) is that traps with the same wall materials have $\tau_{w a 11}$ proportional to $\lambda, i . e .$, proportional to $4 \mathrm{Vol} / A_{w}$. Under the influence of gravity we $f$ ind that this is, in general, only approximately true, although under certain conditions, $\lambda$, as measured along the trajectories, remains equal to $4 \mathrm{Vol} / \mathrm{A}_{\mathrm{w}}$.

This last point is relevant to the determination of the neutron beta decay lifetime from experimental measurements of $\tau_{\text {gross }}$. Values of $\tau_{\text {wall }}$ can not be predicted since there is only very approximate information on $E$ and $f$. Also, it is 
not sure how precisely the form of equation (2) describes the real situation. However, the problem can be circumvented as follows. The minimum requirement is to use two traps labelled 1 and 2 with walls of the same material, but with values of $\tau_{\text {wal1 } 1}$ and $\tau_{\text {wal1 } 2}$ which are arranged to be significantly different by choosing different values of $4 \mathrm{Vol} / A_{H}$. The measured $\tau_{g \text { ross } 1}$ and $\tau_{g r o s s} 2$ for the two traps are obtained using neutrons of same energy in each trap and the provide the two simultaneous equations

$\frac{1}{\tau_{\text {Bross 1 }}}=\frac{1}{\tau_{\text {wa11 1 }}}+\frac{1}{\tau_{n}}$

and

$\frac{1}{\tau_{\text {gross } 2}}=\frac{1}{\tau_{\text {wa11 } 2}}+\frac{1}{\tau_{n}}$.

If the chosen change of trap geometry gives us a simply calculable value for the ratio $a=\left(\begin{array}{lll}\tau_{\text {wall }} & 1\end{array}\right) /\left(\begin{array}{lll}\tau_{\text {wall }} & 2\end{array}\right)$ we can immediately extract the value of $\tau_{n}$. If, for example, in going from trap 1 to trap 2 we halve the value of $4 \mathrm{Vol} / \mathrm{A}_{\mathrm{H}}$ we know from (5) and (6) that in the absence of gravity $a=2$. For this procedure, there is even no need to assume that the loss function has the form of equation (2). In practice, the experiments have to be performed with gravity present. In section 5 we show how this simple approach has then to be modified.

\section{Effective real space volumes with gravity}

Let $\epsilon$ be used for the total energy of the UCN and $E$ and $p$ for their kinetic energy and momentum respectively. The height coordinate $h$ will be defined in the upwards sense relative to a horizontal datum plane $\mathrm{h}=0$ where we will also take the UCN potential energy to be zero. Consequently, under the assumed conditions of elastic wall collisions, $\mathrm{p}^{2} /(2 \mathrm{~m})=\mathrm{E}=\epsilon-$ mgh. We will consider a monoenergetic group of UCN, with energies in the range $\epsilon$ to $\epsilon+\delta \epsilon$ where $\delta \epsilon<\epsilon$. The energies will remain within this same range $\delta \epsilon$ at all times in the future and at all accessible heights. Differentiating $E=\epsilon-m g h$ at any given $h$, shows that the kinetic energies of the group will cover the same magnitude of range $\delta \mathrm{E}=\delta \epsilon$ at all accessible $h$.

To make use of the equilibrium condition that the points representing the UCN will have spread themselves uniformly through the available phase space we need to consider the available momentum space. The range $\delta \mathrm{E}=\delta \epsilon$ implies a range $\delta \mathrm{p}$ where $\delta E=\mathrm{p} \delta \mathrm{p} / \mathrm{m}$ so that the available volume in momentum space may be expressed as

$4 \pi p^{2} \delta p=C \sqrt{E} \delta E=C \sqrt{(\epsilon-m g h)} \delta \epsilon$

where $C=4 \pi \sqrt{2 m^{3}}$. The phase space density for the group is its real space density $n(\epsilon, t, h)$ divided by the volume it occupies in momentum space $C \sqrt{E(h)} \delta \epsilon$. When the 
phase space density has become uniform this ratio is independent of position, and therefore of $h$, so that

$\frac{n(\epsilon, t, h)}{C \sqrt{E(h)} \delta \epsilon}=\frac{n(\epsilon, t, 0)}{C \sqrt{E(0)} \delta \epsilon}$.

Hence

$\frac{n(\epsilon, t, h)}{n(\epsilon, t, 0)}=\sqrt{\frac{E(h)}{E(0)}}=\sqrt{\frac{(\epsilon-m g h)}{\epsilon}}$ when $(\epsilon-m g h)>0$

and $n(\epsilon, t, h)=0$ whenever $(\epsilon-m g h)$ is negative.

We also note that

$\frac{v(\epsilon, h)}{v(\epsilon, 0)}=\sqrt{\frac{(\epsilon-m g h)}{\epsilon}}$

and that

$\frac{n(\epsilon, t, h) v(h)}{n(\epsilon, t, 0) v(0)}=\frac{(\epsilon-m g h)}{\epsilon} \quad$ when $(\epsilon-m g h)>0$

where $\mathrm{nv}$ is set equal to zero whenever $\epsilon-\mathrm{mgh}$ is negative. Equations (11) (12) and (13) can only be applied to a UCN group for which $\delta \epsilon$ is small compared with the changes of mgh in the set-up of interest.

At height $h$ the trap horizontal section area will be called $A(h)$ and the perimeter of this section will be called $P(h)$. The total population $N(\epsilon, t)$ of an energy group in a trap can then be calculated as

$N(\epsilon, t)=\int_{h_{\min }}^{h_{\max }} n(\epsilon, t, h) A(h) d h$

where $h_{\min }$ and $h_{\max }$ are the lowest and highest points in the trap. Using equation (11) this becomes

$N(\epsilon, t)=n(\epsilon, t, 0) \int_{h_{\text {min }}}^{h_{\max }} \sqrt{\frac{(\epsilon-m g h)}{\epsilon}} A(h) d h$

where again the square root function must be replaced by zero whenever its argument is negative. In the absence of gravity this result could have been simply expressed as $N(\epsilon, t)=n(\epsilon, t, 0)$ Vol, so that the integral of equation (15) can be identified as an effective volume $\gamma(\epsilon)$ for the trap under the influence of gravity where

$\gamma(\epsilon)=\int_{h_{\min }}^{h_{\max }} \sqrt{\frac{(\epsilon-\text { mgh })}{\epsilon}} A(h) d h$.

In using (16) to calculate the way neutrons of a group are shared between two 
interconnected traps at different heights the two effective volumes must be calculated using a common datum. If the volume of the connecting pipe can be neglected then the UCN of the group will apportion themselves in the ratio of the two effective volumes. When considering a single trap the effective volume will depend on the choice of datum. Compensating changes in $n(\epsilon, t, 0)$ and in the effective volume ensure that the physical $N(\epsilon, t)$ is not affected by an arbitrary change of datum.

\section{Mean free path along the trajectories in the presence of gravity}

The total rate, $R_{c}$, of making collisions with the walls is given by $R_{c}=\frac{1}{4} \int_{h_{\min }}^{h_{\max }} n(\epsilon, t, h) v(\epsilon, h) P(h) d h$.

The total rate of path making, $R_{p}$, by UCN in the trap is given by $R_{p}=\int_{h_{\min }}^{h_{\max }} n(\epsilon, t, h) v(\epsilon, h) A(h) d h$.

The mean free path $\lambda$ along the trajectories is just $R_{p} / R_{c}$. In the absence of gravity $n$ and $v$ do not vary with position and they can be taken out of the integrals. They then cancel in taking the ratio leaving the clausius result, $\lambda=R_{p} / R_{c}=4 \mathrm{Vol} / A_{w}$.

We find that, under certain conditions, the same result is obtained in the presence of gravity. One of the conditions is that the trap should have a centre of symmetry and the other is that the neutron group should have sufficient energy $\epsilon$ for UCN to be able to reach the highest point of the trap. We now adopt these conditions and put the datum at the level of the centre of symmetry. Equation (13) can now used to express the product nv in (18) in terms of its value at the datum leading to the expression

$R_{c}=\frac{n(\epsilon, t, 0) v(\epsilon, 0)}{4} \int_{-h_{\max }}^{+h_{\max }}\left[1-\frac{\mathrm{mgh}}{\epsilon}\right] P(h) d h$.

The contents of the square bracket would have to be replaced by zero whenever they become negative, but his will never be the case for a neutron group whose members can reach the highest point of the trap. The integrand of (19) is then seen to have two parts. The second part containing mgh is, given the symmetry of the trap, an odd function of $h$ and its integral is therefore zero. The first part is just the total surface area, $A_{W}$. Thus, we have the result $R_{c}=n(\epsilon, t, 0) v(0) A_{w} / 4$. The same methods when applied to $R_{p}$ give $R_{p}=n(\epsilon, t, 0) v(0)$ Vol. The new ratio $R_{p} / R_{c}$ is seen to be equal to $4 \mathrm{Vol} / \mathrm{A}_{\mathrm{w}}$, which is the same as the gravity free result. The most extreme case where this unexpected result applies is that where gravity just 
reduces the UCN number density to zero at the highest point of the trap.

If neutron groups are considered with energies less than that needed to just reach the highest point of the trap, the mean free path falls with total neutron energy until, eventually, it approaches zero at the stage where the paths are tiny parabollic arcs in contact with the lowest element of surface. As a particular example, one might consider a cube of side $\mathrm{L}$ with a horizontal base plane which is taken to be the datum. It is convenient in this problem, and others which follow, to weasure the total neutron energy in terms of the height $\mathrm{H}$ given by $\mathrm{H}=\epsilon / \mathrm{mg}$, which is the greatest height the neutron could rise above the datum against gravity if it was in a trap of unlimited height. Starting from (18) and (19) we obtain

$$
\lambda=\frac{2}{3} L \quad \text { for UCN with } H>L
$$

and

$$
\lambda=\left(\frac{2}{2+L / H}\right) L \text { for UCN with } 0<H<L
$$

as illustrated in fig. 1.

\section{$\mathrm{H} / \mathrm{L}$}

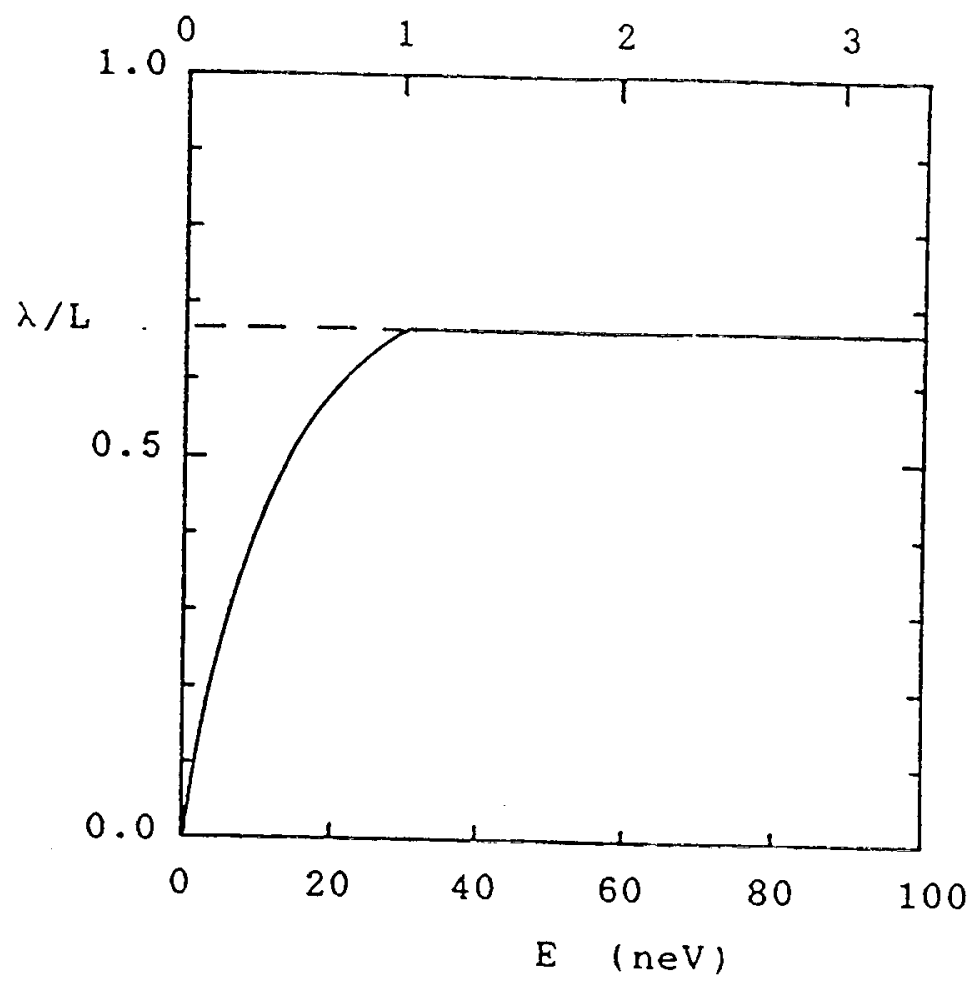

Fig. 1. The mean free path $\lambda$ along the trajectories between wall collisions for a cubic trap of side L, taking into account gravity. The neutron kinetic energy at the base of the trap is $E$ and this is expressed as a height $H=E /(2 g)$. For $H>L$, the value of $\lambda$ is unaffected by gravity and is equal to $2 \mathrm{~L} / 3$. The lower scale showing $E$ in $\mathrm{neV}$ is appropriate to the case $\mathrm{L}=0.3 \mathrm{~m}$. 


\section{Bffect of gravity on losses in wall collisions}

Again we will restrict the discussion to traps with a centre of symmetry and place the datum at the level of the centre. The mean Fermi potential $V$ of the trap wall is represented by the critical height $H_{c}$ where $H_{c}=V / m g$. The UCN total energies are represented by $H$ and at the height $h$ relative to the datum, the UCN kinetic energy is represented by $\mathrm{H}-\mathrm{h}$. Besides the convenience of symmetry, this choice of datum has another advantage. In assessing the modification due to gravity there is a difficulty of comparing like with like concerning the energy of the UCN group with, and without, gravity. For a datum at the lowest point of the trap, equal kinetic energies at the datum means a much lower average kinetic energy in the middle of the trap when gravity is present. This gives a large change in UCN wall loss rate which is mainly expressing the well known feature of UCN that in a given trap slower neutrons suffer a slower rate of loss. If we choose a datum half way up the trap and standardise the kinetic energy of our group there, the difference in the average energy with and without gravity is much smaller. Nevertheless, some ambiguity remains. However, equations (37) and (38) and the gravitational correction which they imply for the neutron lifetime experiments do not depend on the choice of datum except for the way in which the energy labelling of the UCN groups is defined.

The UCN loss rate $d R_{L H}$ on a element of wall of area $d A$ at height $h$ is given by

$\mathrm{dR}_{\mathrm{LH}}=\frac{1}{4} \mathrm{n}(\mathrm{H}, \mathrm{t}, \mathrm{h}) \mathrm{v}(\mathrm{H}, \mathrm{h}) \bar{\mu}\left(H-h, H_{c}\right) \mathrm{dA}$.

For convenience we use (12) and (13) to express this as

$\mathrm{dR}_{\mathrm{LH}}=\frac{1}{4} \mathrm{n}(\mathrm{H}, \mathrm{t}, 0) \mathrm{v}(\mathrm{H}, 0)\left(\frac{\mathrm{H}-\mathrm{h}}{\mathrm{H}}\right) \bar{\mu}\left(\mathrm{H}-\mathrm{h}, \mathrm{H}_{\mathrm{c}}\right)$.

The latter can be written as

$\mathrm{dR}_{\mathrm{LH}}=\frac{1}{4} \mathrm{n}(\mathrm{H}, \mathrm{t}, 0) \mathrm{v}(\mathrm{H}, 0) \mathrm{L}(\mathrm{H}, \mathrm{h})$

where we have introduced the function $L$ defined as

$L(H, h)=\left(\frac{(H-h)}{H}\right) \bar{\mu}\left(H-h, H_{c}\right)$.

For the total loss rate we have

$R_{L H}=\frac{1}{4} n(H, t, 0) v(H, 0) \int_{-h_{\max }}^{+h_{\max }} L(H, h) P(h) d h$.

From (15) the total number of stored neutrons in this energy group at time $t$ is

$N(H, t)=n(H, t, 0) \int_{-h_{\max }}^{+h_{\max }} \sqrt{\frac{(H-h)}{H}} A(h j d h$. 
These expressions can be used to evaluate $\tau_{\text {wall }}$ using

$\tau_{\text {wa11 }}=\frac{N(H, t)}{R_{L H}}$

which is seen to be independent of $t$ giving a pure exponential decay.

The only approximation up to this point has come from ignoring the very small anisotropies in the neutron velocities, referred to earlier, which can be as little as 1 part in $10^{3}$. However, before considering intergrals over $h$, we now choose to introduce a Taylor series expansion of the function $\mathrm{L}$ in terms of the variable $h / H_{c}$. In fact, the integrals over $L$ can be done directly without making any expansion [9]. While this is useful for exact numerical results, we feel the series expansion method is more helpful here for understanding some features of this system. Therefore, we use

$L\left(H, h / H_{c}\right)=L(H, 0)\left[1+a_{1}\left(\frac{h}{H_{c}}\right)+a_{2}\left(\frac{h}{H_{c}}\right)^{2}+a_{3}\left(\frac{h}{H_{c}}\right)^{3} \ldots\right]$

where the second coefficient, $a_{2}$, for example, is given by

$a_{2}=\frac{L^{\prime \prime}(H, 0)}{2 L(H, 0)}$

and the double prime represents differentiation with respect to the dimensionless variable $\mathrm{h} / \mathrm{H}_{\mathrm{c}}$. In the Fomblin oil coated trap of Mampe et al [3] the maximum value of $\mathrm{h} / \mathrm{H}_{\mathrm{c}}$, which was reached at the trap roof, was 0.14 . Inserting (29) into the integral (26) we have

$R_{L H}=\frac{1}{4} n(H, t, 0) v(H, 0) L(H, 0) \int_{-h_{\max }}^{t h_{\max }}\left[1+a_{1}\left(\frac{h}{H_{c}}\right)+a_{2}\left(\frac{h}{H_{c}}\right)^{2} \ldots\right] P(h) d h$.

The leading term simply gives the loss rate expression which one would have for this energy group in the absence of gravity. For the subsequent terms in the expansion, we observe that, for the given trap symmetry and datum, all terms with odd powers of $\mathrm{h} / \mathrm{H}_{\mathrm{c}}$ will contribute nothing to the integral. Thus, the largest term in the loss rate per neutron which has any dependence on gravity comes from the $\left(\mathrm{h} / \mathrm{H}_{\mathrm{c}}\right)^{2}$ term. Equation $(30)$ shows how the ratio, between the curvature and the value of the loss function $L$, gives the value of the coefficient $a_{2}$. In fig. 2 we show $a_{2}$ as a function of the energy $H$ of the UCN group in the case where the ideal form of $\bar{\mu}$ given in equation (2) applies. The value of $a_{2}$ is smallest and changing rather slowly with the UCN energy at intermediate energies. When dealing with the collision rate, as in calculating the mean free path, the height variation was linear. When calculating loss rates we are dealing with collision rate multiplied by the loss per collision. The latter is also changing with height since the collisions are more violent at lower levels; the result is that loss rates have a non-linear dependence on $h$. 


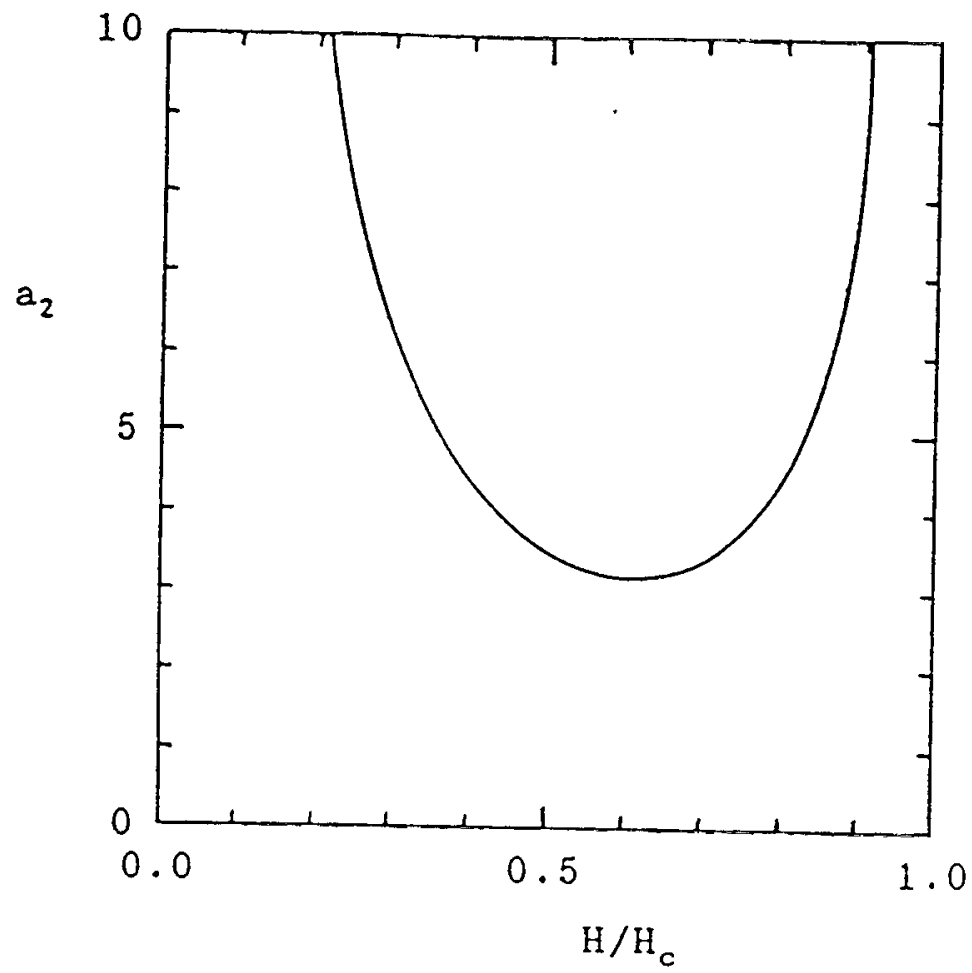

Fig. 2. The dimensionless coefficient $a_{2}$ in the leading term of the modification due to gravity of the wall loss rate for UCN which can reach the highest point of a trap which has a centre of symmetry. It has been calculated according to equations (30) and (25) using the ideal wall loss function (2). It is plotted as a function of the ratio of the UCN energy to the critical energy of the wall material. 


\section{UCN $108 s$ modifications for rectangular traps}

Considering now a rectangular trap with a horizontal floor, let the combined area of the roof and floor be $2 A_{\mathrm{g}}$ and the area of the side-walls be $A_{S}$. The integral (31) for the rate of loss on the vertical walls gives

$$
R_{L H S}=\frac{1}{4} n(H, t, 0) v(H, 0) L(H, 0) A_{S}\left[1+\frac{a_{2}}{3}\left(\frac{h_{t}}{2 H_{c}}\right)^{2} \ldots\right]
$$

where $h_{t}$ is the height of the trap. Converting (32) back to normal units gives

$$
R_{L \text { w }}=\frac{1}{4} n(\epsilon, t, 0) v(\epsilon, 0) \bar{\mu}(\epsilon, 0) A_{S}\left[1+\frac{a_{2}}{3}\left(\frac{m g h_{t}}{2 v}\right)^{2} \ldots\right]
$$

which shows that $g$ is only present in the $a_{2}$ term and higher order terms. The coefficient $a_{2}$ is dimensionless and does not change in this conversion. For the trap of reference [3] where $h_{t}=0.3 \mathrm{~m}$ the term $\left(a_{2} / 3\right)\left(\frac{1}{2} m g h_{t} / V\right)^{2}$ was equal to 0.02 . In the case of the roof and the floor the integrand of (31) is only finite at $\pm h_{\max }$ and we have

$$
R_{L H F}=\frac{1}{4} n(H, t, 0) v(H, 0) L(H, 0) 2 A_{F}\left[1+a_{2}\left(\frac{h_{t}}{2 H_{c}}\right)^{2} \ldots\right] \text {. }
$$

Adding (32) and (34) to obtain the total losses and introducing the total trap wall area $A_{W}=A_{S}+2 A_{F}$ gives

$R_{L H}=\frac{1}{4} n(H, t, 0) v(H, 0) L(H, 0) A_{w}\left[1+\frac{a_{2}}{3}\left(\frac{h_{t}}{2 H_{c}}\right)^{2}\left(1+\frac{4 A_{F}}{A_{w}}\right) \cdots\right]$.

The number of stored UCNs given by (28) can be integrated and expanded binomially to give

$N(H, t)=n(H, t, 0) \operatorname{Vol}\left[1-\frac{1}{24}\left(\frac{h_{t}}{2 H}\right)^{2} \ldots\right]$

where Vol is the true volume of the trap. The expression for $\tau_{W a 11}^{-1}$ for the UCN group is obtained from (35) divided by $(36)$. At the same time we replace $A_{F}$ by $\mathrm{Vol} / \mathrm{h}_{\mathrm{t}}$ and use the fact that $4 \mathrm{Vol} / \mathrm{A}_{\mathrm{F}}=\lambda$ to find

$\tau_{w a 11}^{-1}=\frac{v(H, 0)}{\lambda} L(H, 0)\left[1+\frac{a_{2}}{3}\left(\frac{h_{t}}{2 H_{c}}\right)^{2}\left(1+\frac{\lambda}{h_{t}}\right) \ldots\right]\left[1+\frac{1}{24}\left(\frac{h_{t}}{2 H}\right)^{2} \ldots\right]$.

A rearrangement then gives 


$$
\begin{aligned}
\tau_{w a 11}^{-1}=\left\{\frac{v(H, 0) L(H, 0)}{h_{t}}\left[\frac{a_{2}}{3}\left(\frac{h_{t}}{2 H_{c}}\right)^{2}+\ldots\right]\right. \\
\left.\left.\quad+\frac{v(H, 0) L(H, 0)}{\lambda}\left[1+\frac{a_{2}}{3}\left(\frac{h_{t}}{2 H_{c}}\right)^{2}\right) \ldots\right]\right\}\left[1+\frac{1}{24}\left(\frac{h_{t}}{2 H}\right)^{2} \ldots\right] .
\end{aligned}
$$

The next term in the first series in (37) is proportional to $a_{4}$ and contains the factor $\left(1+2 \lambda / h_{t}\right)$ and so on. At this point it becomes evident that the only $\lambda$ dependence in (38) is that which is shown explicitly. Thus, the form of (38) is $\tau_{w a 11}^{-1}=A+B \lambda^{-1}$ and we can write $\tau_{B \text { ross }}^{-1}=\tau_{n}^{-1}+A+B \lambda^{-1}$.

The conclusion is that if a particular UCN energy group is stored in a series of traps, all with the same wall material the same height $h_{t}$, but with various widths and volumes, the wall loss rates, as represented by the $\tau_{w a 11}^{-1}$ values, have a major part proportional to the reciprocal mean free path $\lambda^{-1}$ but, added to this, there is a small constant, $A$, given by that part of (38) which is on the first line, where coefficients such as $a_{2}$ are defined by $(29)(30)(25)$ and (2) and the whole is multiplied by the effective volume factor at the end of (38). To see the size of $A$, we take as an example, the conditions of the experiments in reference [3] where $h_{t}=0.30 \mathrm{~m}$ and $H_{c}=1.04 \mathrm{~m}$. Then, for the UCN group with $H=0.7 H_{c}$, using the function (2) with $\mathrm{f}=\mathrm{W} / \mathrm{V}=1.6 \times 10^{-5}$, we obtain $\mathrm{A}=5.7 \times 10^{-6} \mathrm{~s}^{-1}$. In the neutron lifetime experiments the measured $\tau_{g r o s s}^{-1}$ for different trap lengths are plotted against $\lambda^{-1}$ and extrapolated to obtain the intercept. (In the case of just two measurements with two trap lengths, the intercept can be found by solving simultaneous equations as mentioned in section 1 ). The intercept at $\lambda^{-1}=0$, is the sum of $\tau_{n}^{-1}$ and the constant $A$. The latter must therefore be subtracted for estimating $\tau_{n}^{-1}$. For the size of A just calculated, the subtraction causes a shift of $5 \mathrm{~s}$ or $0.5 \%$ in the result for $\tau_{n}$. This shift has become known as the gravity correction. It is interesting how small the correction is, since the terms from the second line of (38) denoted $B \lambda^{-1}$, for the case where $\lambda=h_{t}$, represent wall losses which are about $15 \%$ of $\tau_{n}^{-1}$. Another way to view the result (38) is, that for the purpose of calculating wall losses, $\lambda^{-1}$ has a small constant of set of $A / B$ added to it.

The experiments in [3] used a broad UCN energy spectrum so that this correction took the form of a weighted average over the spectrum. A more complete computer model was built using the methods outlined here applied to a large number of UCN energy groups. In the model the integrals were done numerically and the Taylor series expansion was not needed. Nevertheless, the estimated correction was similar in size to that just evaluated. Furthermore, the computer model confirmed that the correction does not change if the choice of height for the datum is 
changed.

Although this correction in the $\tau_{n}$ measurements is small, it changes the situation in a fundamental way. If $\tau_{n}$ is only measured to a precision of $1 \%$ the correction can be ignored, in which case the experimental data can be processed using nothing more than the Clausius mean free paths (6). The form of the $\bar{\mu}(E)$ function does not need to be considered and the only thing required of the UCN energy spectrum is that all UCN should be capable of reaching the top of the trap and that the shape of the spectrum after filling should be independent of the trap geometry. However, it now seems feasible and desirable to try to measure $\tau_{n}$ to a precision of $0.1 \%$. In this case the $0.5 \%$ gravity correction has to be evaluated quite carefully. This, in turn, requires some knowlege of the shape of the $\bar{\mu}(E)$ function $[7,8,9]$ and also some knowledge of the energy spectrum of the stored UCN.

\section{Hays of reducing the gravity correction}

The equations above allow one to see ways of largely eliminating this correction. The ideal situation when measuring $\tau_{n}$ would be to have the rate of loss of stored UCN per unit area of surface be the same for all parts of the trap surface. In the absence of gravity this can be achieved easily provided we have a reproducible surface material such as a clean liquid. In the presence of gravity the correction results from the following leature: the average loss per unit area on the roof and floor combined is a little greater than the average loss per unit area on the vertical side-walls. In the experiments of reference [3] $\lambda^{-1}$ is changed by advancing a plane vertical wall as shown in fig. 3(a). With the advance, the ratio of roof plus floor area, to side-wall area, reduces. This causes the overall trap average wall loss per unit area to fall slightly whereas we would like the wall quality to stay constant. A way to avoid this change in relative areas is shown in fig. $3(\mathrm{~b})$. The profile shown is maintained across the full width of the trap. The trap now has two recesses which increase the amount of roof and floor area. Proceeding under the assumption that the upper and lower surfaces of the upper recess are both nearly enough at the height of the roof, then for the case where the fixed width of the trap, perpendicular to the plane of $\mathrm{fig} .3(\mathrm{~b})$ is equal to the height of the trap, the length $x$ of the recess should be set equal to $h_{t} / 2$. Continuing to neglect the height of the recesses, the total combined roof and floor area for the entire trap is now equal to the total side-wall area.

Furthermore, this remains true for all positions of the movable wall. Thus, the desired constancy of the ratio of areas is achieved. For this particular case, the term $1+4 A_{F} / A_{H}$ in (35) which became $1+\lambda / h_{t}$ in (37) becomes 3 in both equations. There are similar replacements in all such terms in the series of (37) and one can conclude that $\tau_{\text {wal1 }}^{-1}$ is proportional to $\lambda^{-1}$ with no added constant. 
(a)

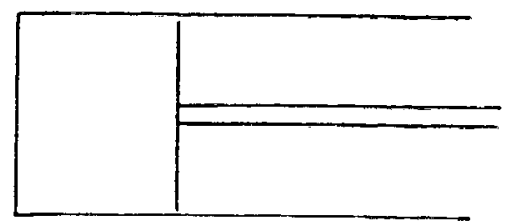

$\rightarrow \times 5$

(b)

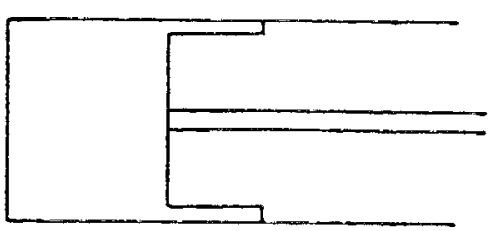

(c)

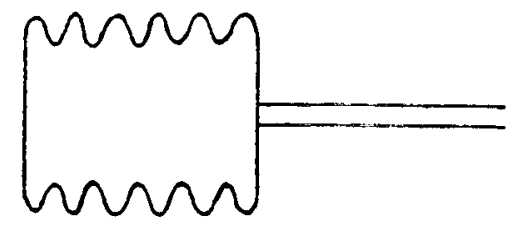

Fig. 3. Three kinds of trap where the mean free path between wall collisions can be varied by changing the trap length. (a) represents the trap used in the neutron lifetime measurement [3]. (b) and (c) show traps where the gravity correction arising in [3] would be largely eliminated. In case (b) for traps where the height is equal to the fixed width perpendicular to the figure, the length $x$ should be half the height of the trap. Case (c) is a trap made with a bellows. 
Another approach which will largely eliminate the correction is to use a trap in the form of a bellows with its axis horizontal as represented by $\mathrm{fig}$. $4 \mathrm{c}$. When the bellows is compressed, the volume reducess while the surface remains the unchanged. It is also evident that the distribution of the wall area with respect to vertical position remains the unchanged to first order. The corrugations of the bellows which occur in various planes would be excellent for maintaining the isotropy of the UCN velocities. On disadvantage of the bellows is the increased wall area which brings increased wall losses in relation to the volume.

\section{Acknowledgenents}

We would like to express our appreciation for stimulating discussions with S.M. Burnett, V. Nesvizhevsky, F. Schorr and also W. Mampe whose untimely death is a great loss to the field. We also thank P. Ageron, A. Robert and A. Martinson for informing us of their Monte Carlo calculations which were in agreement with some of the results obained in this paper. We thank Institut Laue-Langevin for financial support for DJR at the time this work was done.

\section{References}

[1] V.I. Morozov, Nucl. Instr. and Meth. A284 (1989) 108-15.

[2] Sov. Phys. JETP 75 (1992) 405-12.

[3] W. Mampe, P. Ageron, J.C. Bates, J.M. Pendlebury and A. Steyerl, Nucl Inst. and Meth. A284 (1989) 111-5 \& Phys. Rev. Letts. 63 (1989) 593-6.

[4] V.I. Morozov et al, JETP Letts, 57 (1993) 82-87.

[5] R. Golub and J.M. Pendlebury, Rep. Prog. Phys. 42 (1979) 439-501.

[6] R. Golub, D.J. Richardson and S.K. Lamoreaux, Ultra-cold Neutrons (Adam Hilger, Bristol 1991).

[7] M. Chouder, J.M. Pendlebury and K.F. Smith, Nucl. Instrum. Meths. A311 (1992) 287-292.

[8] D.J. Richardson et al., Nucl. Instr, and Meth. A308 (1991) 568-73.

[9] V.K. Ignatovich, The Physics of Ultracold Neutrons, pp. 211-3 (Clarendon, Oxford 1990). 